\title{
15 THE EFFECTIVENESS OF EMPLOYING EXCLUSIVE ADVANCE CARE PLANNING (ACP) CLINICIANS
}

K Wallis ${ }^{1}$, K Detering ${ }^{1}$, K Whiteside ${ }^{1}$, D Mawren', W Silvester ${ }^{1}{ }^{1}$ Respecting Patient Choices Program Austin Health

10.1136/bmjspcare-2012-000250.15

ACP improves end-of-life care and patient/family satisfaction. Nevertheless, health professionals struggle to include ACP in their busy work schedule in a university hospital environment. In response, since 2008, the Respecting Patient Choices Program has employed nurses exclusively to do ACP. A prospective audit of the activity of these "ACP Clinicians" was conducted 1/1/2010 - 31/12/2011. The audit included patient demographics, reason for referral and ACP activity and outcomes. 1866 patients (median age 76 (18-110), 51\% male) were referred. Their primary admission diagnoses were cancer $23 \%$ or renal- $18 \%$, respiratory- $12 \%$ or cardiac- $10 \%$ failure. The main reasons for referral were age/debility/multiple co-morbidities or poor prognosis. Prior to referral $11.5 \%$ of patients had previously appointed a substitute decision maker (SDM), and 5\% had completed an advance care plan. 1703/1866 (91\%) referred patients were seen by an ACP Clinician. Of these $45 \%$ of patients completed ACP documentation: SDM alone $(33 \%)$, advance care plan alone $(11 \%)$ or a combination of both $(56 \%)$. If documents were completed, the time spent per patient was longer (median 90 mins vs. 45 mins) and the number of visits were more (median 2 vs. 1). When patients completed an advance care plan outlining treatment preferences, $56 \%$ expressed a wish to not receive cardiopulmonary resuscitation, $45 \%$ did not want life-prolonging treatment at all, $45 \%$ only wanted treatment if a patient-defined reasonable outcome was expected, and a further $7 \%$ delegated the decision to others. Employment of ACP clinicians is an effective method of providing ACP, enabling patients to complete documents if desired. 